TRANSACTIONS OF THE

AMERICAN MATHEMATICAL SOCIETY

Volume 357, Number 8, Pages 3299-3310

S 0002-9947(04)03605-0

Article electronically published on December 28, 2004

\title{
GENERATING THE SURFACE MAPPING CLASS GROUP BY TWO ELEMENTS
}

\author{
MUSTAFA KORKMAZ
}

\begin{abstract}
Wajnryb proved in 1996 that the mapping class group of an orientable surface is generated by two elements. We prove that one of these generators can be taken as a Dehn twist. We also prove that the extended mapping class group is generated by two elements, again one of which is a Dehn twist. Another result we prove is that the mapping class groups are also generated by two elements of finite order.
\end{abstract}

\section{INTRODUCTION}

Let $\Sigma$ be a compact connected oriented surface of genus $g$ with one boundary component. We denote by $\operatorname{Mod}_{g}^{1}$ the mapping class group of $\Sigma$, the group of isotopy classes of orientation-preserving diffeomorphisms $\Sigma \rightarrow \Sigma$ which restrict to the identity on the boundary. The isotopies are also required to fix the points on the boundary. If the diffeomorphisms and the isotopies are allowed to permute the points on the boundary of $\Sigma$, then we get the group $\operatorname{Mod}_{g, 1}$. The extended mapping class group $\operatorname{Mod}_{g, 1}^{*}$ of $\Sigma$ is defined to be the group of isotopy classes of all (including orientation-reversing) diffeomorphisms of $\Sigma$. These three groups are related to each other as follows: $\operatorname{Mod}_{g, 1}$ is contained in $\operatorname{Mod}_{g, 1}^{*}$ as a subgroup of index two and the groups $\operatorname{Mod}_{g}^{1}$ and $\operatorname{Mod}_{g, 1}$ fit into a short exact sequence

$$
1 \rightarrow \mathbb{Z} \rightarrow \operatorname{Mod}_{g}^{1} \rightarrow \operatorname{Mod}_{g, 1} \rightarrow 1
$$

where $\mathbb{Z}$ is the subgroup of $\operatorname{Mod}_{g}^{1}$ generated by the Dehn twist about a simple closed curve parallel to the boundary component of $\Sigma$.

In this paper, we will be interested in the $\operatorname{groups}_{\operatorname{Mod}}^{1}, \operatorname{Mod}_{g, 1}$ and $\operatorname{Mod}_{g, 1}^{*}$. The mapping class group and the extended mapping class group of the closed surface of genus $g$ obtained from $\Sigma$ by gluing a disc along the boundary component are denoted by $\operatorname{Mod}_{g}$ and $\operatorname{Mod}_{g}^{*}$.

The mapping class group is a central object in low-dimensional topology. Therefore, its algebraic structures are of interest. The problem of finding a set of generators for the mapping class group of a closed orientable surface was first considered by Dehn. He proved in [2] that $\operatorname{Mod}_{g}$ is generated by a finitely many Dehn twists. About quarter century later, Lickorish [5, 6] also proved the same result, showing that $3 g-1$ Dehn twists generate $\operatorname{Mod}_{g}$. This number was improved to $2 g+1$ by Humphries [3]. These $2 g+1$ generators are the Dehn twists about the curves

Received by the editors November 8, 2003 and, in revised form, January 8, 2004.

2000 Mathematics Subject Classification. Primary 57M07, 20F05; Secondary 57M60, 20 F38.

The author was supported in part by the Turkish Academy of Sciences under the Young Scientists Award Program (MK/TÜBA-GEBIP 2003-10). 
$b, a_{1}, a_{2}, \ldots, a_{2 g}$ in Figure 1, where the closed surface is obtained from $\Sigma$ by gluing a disc along the boundary component. Humphries proved, moreover, that in fact the number $2 g+1$ is minimal; i.e. $\operatorname{Mod}_{g}$ cannot be generated by $2 g$ (or less) Dehn twists. Johnson [4] proved that the $2 g+1$ Dehn twists about $b, a_{1}, a_{2}, \ldots, a_{2 g}$ on $\Sigma$ also generate $\operatorname{Mod}_{g}^{1}$. Finally, the minimal number of generators for the mapping class group is determined by Wajnryb 8]. He showed that $\operatorname{Mod}_{g}^{1}$, and hence $\operatorname{Mod}_{g}$, can be generated by two elements; one is a product of two Dehn twists (one is right and one is left) and the other is a product of $2 g$ Dehn twists. Since the mapping class group is not abelian, the number two is minimal. Recently, it was shown by Brendle and Farb in [1] that the mapping class group is generated by three torsion elements and by six involutions.

Since $\operatorname{Mod}_{g, 1}$ is a quotient of $\operatorname{Mod}_{g}^{1}$, it is generated by the corresponding $2 g+1$ Dehn twists. In order to generate the extended mapping class group $\operatorname{Mod}_{g, 1}^{*}$, it suffices to add one more generator, namely the isotopy class of any orientationreversing diffeomorphism.

In this paper we have three main results. First, we improve Wajnryb's result. We show that one of the two generators of $\operatorname{Mod}_{g}^{1}$ can be taken as a Dehn twist. All Dehn twists involved in our generators are Dehn-Lickorish-Humphries generators, as opposed to Wajnryb's generators. We also prove that the extended mapping class group $\operatorname{Mod}_{g, 1}^{*}$ is generated by two elements, again one of which is a Dehn twist. Our proofs are independent from that of Wajnryb [8]. Next, we prove that the mapping class groups $\operatorname{Mod}_{g, 1}$ and $\operatorname{Mod}_{g}$ are also generated by two torsion elements. Of course, this is not true for $\operatorname{Mod}_{g}^{1}$ since it is torsion-free. In the last section of the paper, we transform the presentation of the mapping class group in 7 into a presentation on our two generators.

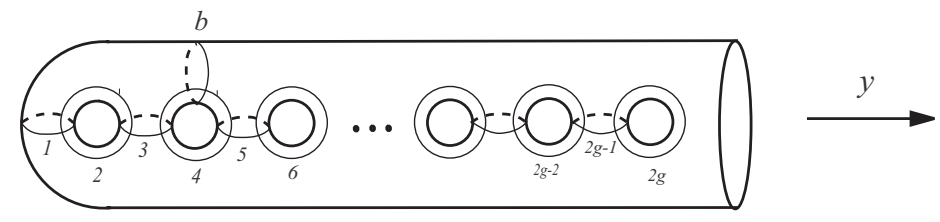

Figure 1. The curve labelled $i$ is $a_{i}$.

\section{Preliminaries}

Recall that if $a$ is a simple closed curve on the oriented surface $\Sigma$, then the (right) Dehn twist $A$ about $a$ is the isotopy class of the diffeomorphism obtained by cutting $\Sigma$ along $a$, twisting one of the side by $2 \pi$ to the right and gluing two sides of $a$ back to each other. We denote the curves by the letters $a, b, c, d$ (possibly with subscripts) and the Dehn twists about them by the corresponding capital letters $A, B, C, D$. Notationally we do not distinguish a diffeomorphism/curve and its isotopy class.

We use the functional notation for the composition of two diffeomorphisms; if $F$ and $G$ are two diffeomorphisms, then the composition $F G$ means that $G$ is applied first. 
We define the curves $c_{i}, d_{i}, \bar{c}_{i}$ and $\bar{d}_{i}$ on $\Sigma$ as shown in Figure 2 so that $\bar{c}_{i}$ (resp. $\bar{d}_{i}$ ) is obtained from $c_{i}$ (resp. $d_{i}$ ) by rotating the surface $\Sigma$ about the $y$-axis by $\pi$. These curves will be used throughout the paper. Here, we assume that the surface of the paper is the $y z$-plane, the positive side of the $x$-axis is pointing above the page and the surface is invariant under the rotation by $\pi$ about the $y$-axis.
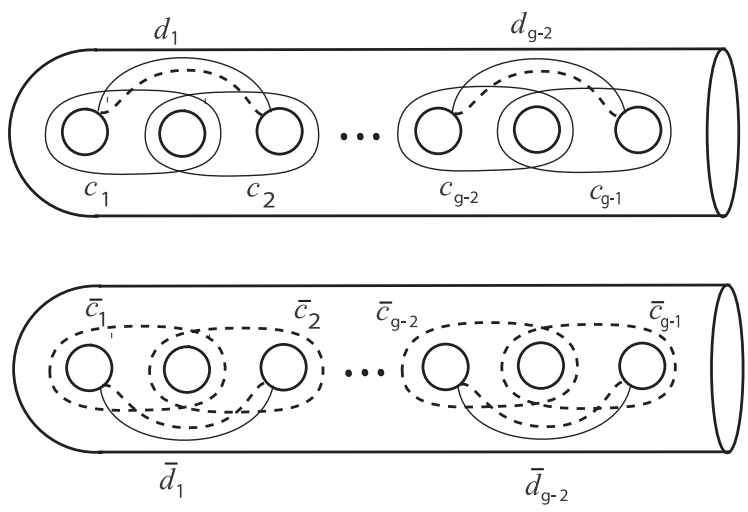

Figure 2. The curves $c_{i}, d_{i}, \bar{c}_{i}$ and $\bar{d}_{i}$.

Let $G$ be a subgroup of $\operatorname{Mod}_{g}^{1}$ or $\operatorname{Mod}_{g, 1}^{*}$. Then $G$ acts on the set of the isotopy classes of simple closed curves. If $c$ is a simple closed curve, then we denote by $c^{G}$ the $G$-orbit of $c$;

$$
c^{G}=\{F(c): F \in G\} .
$$

We record the following lemmas.

Lemma 2.1. Let $c$ be a simple closed curve on $\Sigma$, let $F$ be a self-diffeomorphism of $\Sigma$ and let $F(c)=d$. Then $F C F^{-1}=D^{r}$, where $r= \pm 1$ depending on whether $F$ is orientation-preserving or orientation-reverving.

Lemma 2.2. Let $c$ and $d$ be two simple closed curves on $\Sigma$. If $c$ is disjoint from $d$, then $C D=D C$.

Lemma 2.3. Let $c$ and $d$ be two simple closed curves on $\Sigma$. Suppose that $C \in G$ and the curve $d$ is contained in $c^{G}$. Then $D$ is also contained in $G$.

Proof. Since $d \in c^{G}, F(c)=d$ for some $F \in G$. By Lemma 4.1, $F C F^{-1}=D^{r}$. This shows that $D$ is contained in $G$.

\section{The MaPping Class Group $\operatorname{Mod}_{g}^{1}$}

Let $S$ denote the product $A_{2 g} A_{2 g-1} \cdots A_{2} A_{1}$ of $2 g$ Dehn twists in $\operatorname{Mod}_{g}^{1}$ and let $G$ be the subgroup of $\operatorname{Mod}_{g}^{1}$ generated by $B$ and $S$. We prove in this section that $G=\operatorname{Mod}_{g}^{1}$. It follows that the mapping class groups $\operatorname{Mod}_{g, 1}$ and $\operatorname{Mod}_{g}$ are also generated by $B$ and $S$. The main idea of the proof is to show that the $G$-orbit $b^{G}$ of the curve $b$ contains the simple closed curves $a_{1}, a_{2}, \ldots, a_{2 g}$.

Lemma 3.1. The curves $c_{1}, c_{2}, \ldots, c_{g-1}$ and $d_{1}, d_{2}, \ldots, d_{g-2}$ of Figure 2 are contained in $b^{G}$, the $G$-orbit of $b$. 
Proof. This follows from these easily verified facts: The diffeomorphism $S^{-1}$ maps

- $b$ to $c_{1}$,

- $c_{i}$ to $d_{i}$, and

- $d_{i}$ to $c_{i+1}$.

Remark. It can be shown that $S^{2 g+1}\left(c_{i}\right)=\bar{c}_{i}$ and $S^{2 g+1}\left(d_{i}\right)=\bar{d}_{i}$, so that $\bar{c}_{i}$ and $\bar{d}_{i}$ are also in $b^{G}$. These facts will be used in Section 5 in the proof of the fact that the mapping class groups $\operatorname{Mod}_{g, 1}$ and $\operatorname{Mod}_{g}$ are generated by two torsion elements.

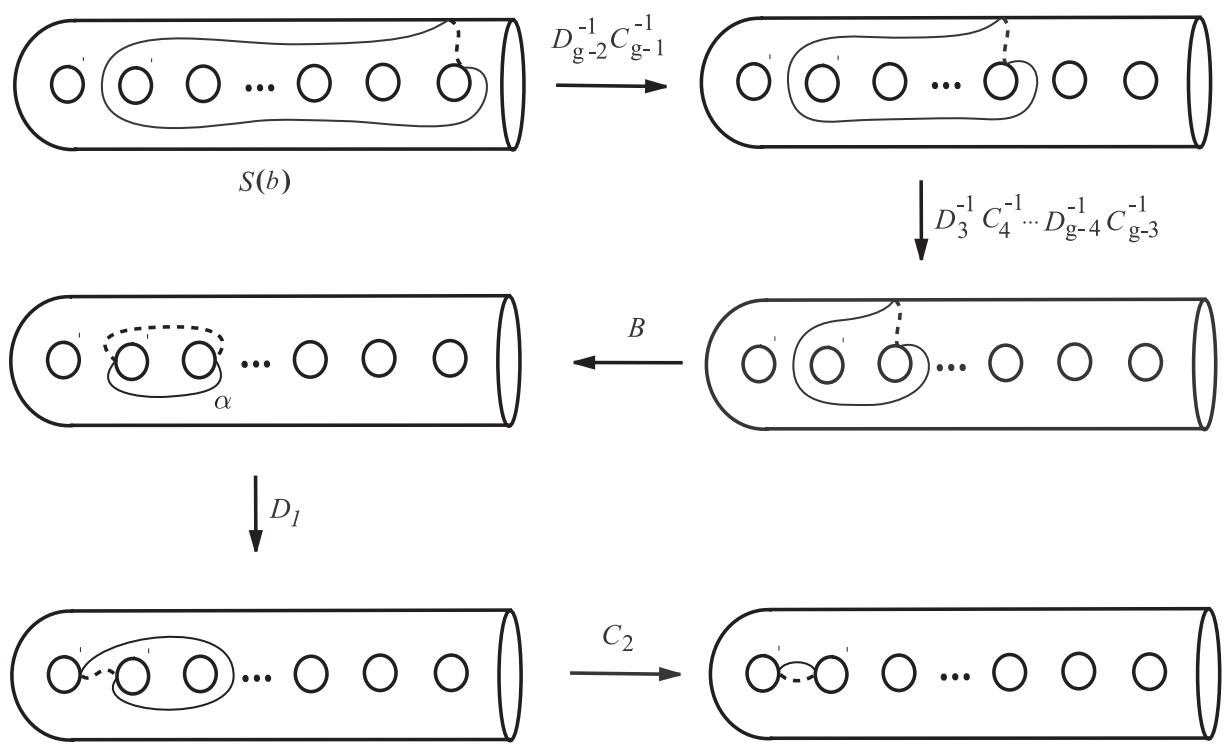

FIGURE 3. $g$ is odd.

Theorem 3.2. Suppose that $g \geq 2$. The subgroup $G$ generated by $B$ and $S$ is equal to the mapping class group $\operatorname{Mod}_{g}^{1}$.

Proof. It can easily be shown that $S\left(a_{i}\right)=a_{i-1}$. Hence $S A_{i} S^{-1}=A_{i-1}$, and thus $A_{i-1} \in G$ if and only if $A_{i} \in G$.

Suppose that the curve $a_{i_{0}}$ is contained in $b^{G}$ for some $i_{0}$. Since $B \in G$, by Lemma 2.3 we get that $A_{i_{0}}$ is also contained in $G$. Therefore, all $A_{i}$ are contained in $G$. Since the mapping class group $\operatorname{Mod}_{g}^{1}$ is generated by the $2 g+1$ Dehn twists $A_{1}, A_{2}, \ldots, A_{2 g}$ and $B$, we conclude that the subgroup $G$ is in fact equal to $\operatorname{Mod}_{g}^{1}$. Therefore, in order to finish the proof of the theorem it suffices to show that $a_{i}$ is contained in $b^{G}$ for some $i$ with $1 \leq i \leq 2 g$.

Suppose first that $g$ is odd. It is easy to see that the diffeomorphism

$$
B D_{3}^{-1} C_{4}^{-1} D_{5}^{-1} C_{6}^{-1} \cdots D_{g-2}^{-1} C_{g-1}^{-1}
$$

maps the curve $S(b)$ to a curve $\alpha$ and $C_{2} D_{1}$ maps $\alpha$ to $a_{3}$ (cf. Figure 3). Since all $C_{i}$ and $D_{i}$ are in $G$, the curve $a_{3}$ is contained in the $G$-orbit $b^{G}$ of $b$.

Suppose now that $g$ is even. In this case the diffeomorphism

$$
B D_{2}^{-1} C_{3}^{-1} D_{4}^{-1} C_{5}^{-1} \cdots D_{g-2}^{-1} C_{g-1}^{-1}
$$


maps the curve $S(b)$ to $a_{4}$. Again, since all $C_{i}$ and $D_{i}$ are in $G$, we conclude that $a_{4}$ is contained in $b^{G}$.

This concludes the proof of the theorem.

\section{The EXTEnded MAPPING ClASS GROUP $\operatorname{Mod}_{g, 1}^{*}$}

In this section we prove that the extended mapping class $\operatorname{group}_{\bmod }^{*}$ is also generated by two elements, one of which is a Dehn twist.

Consider the surface $\Sigma$ embedded in the 3 -space as shown in Figure 1, Let $R$ denote the reflection across the $x y$-plane and let $T$ denote the product $A_{2 g} \cdots$ $A_{3} A_{2} A_{1} R$. Let $H$ denote the subgroup of $\operatorname{Mod}_{g, 1}^{*}$ generated by $B$ and $T$. We prove that $H=\operatorname{Mod}_{g, 1}^{*}$. Again, it follows that $\operatorname{Mod}_{g}^{*}$ is also generated by $B$ and $T$. Recall that the $H$-orbit of the simple closed curve $b$ is denoted by $b^{H}$.

Lemma 4.1. (1) If $g$ is even, then the curves $c_{1}, \bar{c}_{2}, c_{3}, \bar{c}_{4}, \ldots, \bar{c}_{g-2}, c_{g-1}$ and $\bar{d}_{1}, d_{2}, \bar{d}_{3}, d_{4}, \ldots, \bar{d}_{g-3}, d_{g-2}$ of Figure 2 are contained in $b^{H}$.

(2) If $g$ is odd, then the curves $c_{1}, \bar{c}_{2}, c_{3}, \bar{c}_{4}, \ldots, c_{g-2}, \bar{c}_{g-1}$ and $\bar{d}_{1}, d_{2}, \bar{d}_{3}, d_{4}, \ldots$, $d_{g-3}, \bar{d}_{g-2}$ of Figure 2 are contained in $b^{H}$.

Proof. It can be shown easily that the diffeomorphism $T^{-1}$ maps

- $b$ to $c_{1}$,

- $c_{i}$ to $\bar{d}_{i}$,

- $\bar{c}_{i}$ to $d_{i}$,

- $d_{i}$ to $c_{i+1}$, and

- $\bar{d}_{i}$ to $\bar{c}_{i+1}$.

The lemma follows from these.

Lemma 4.2. Suppose that $g$ is odd. Then the curves $e_{1}, e_{2}, e_{3}$ and $e_{4}$ in Figure 4 are contained in $b^{H}$.
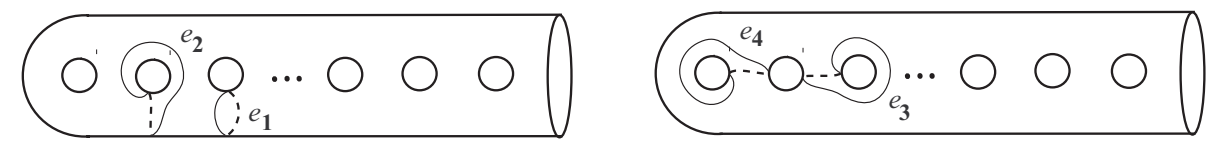

Figure 4 . The curves $e_{i}$.

Proof. Let $U_{i}$ denote $\left(\bar{C}_{i}\right)^{-1}\left(\bar{D}_{i+1}\right)^{-1}$. If $i$ is even, then $U_{i}$ is contained in $H$ by Lemma 4.1 and Lemma 2.3. Now the diffeomorphism

$$
U_{2} U_{4} U_{6} \cdots U_{g-3}\left(\bar{C}_{g-1}\right)^{-1} T
$$

is contained in $H$ and it maps the curve $b$ to $e_{1}$ (cf. Figure 5). This proves that $e_{1} \in b^{H}$.

For the proof of $e_{2} \in b^{H}$ let $U$ denote the diffeomorphism

$$
\bar{C}_{2} D_{2} \bar{C}_{4} D_{4} \cdots \bar{C}_{g-3} D_{g-3} .
$$

Now it suffices to show that

$$
C_{1} U \bar{C}_{g-1} T^{-1}
$$

maps the curve $\bar{c}_{g-1}$ to $e_{2}$. This can be seen from Figure 6 


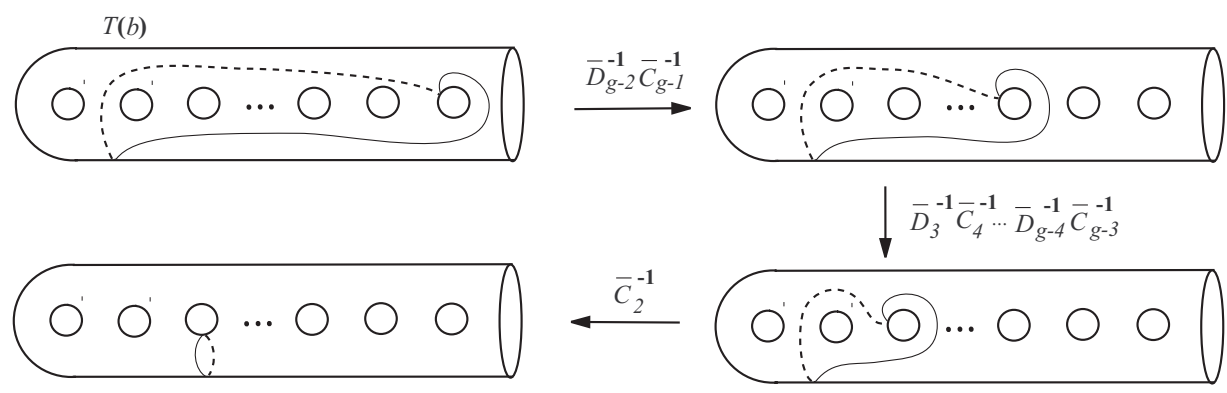

Figure 5. The proof of $e_{1} \in b^{H}$.

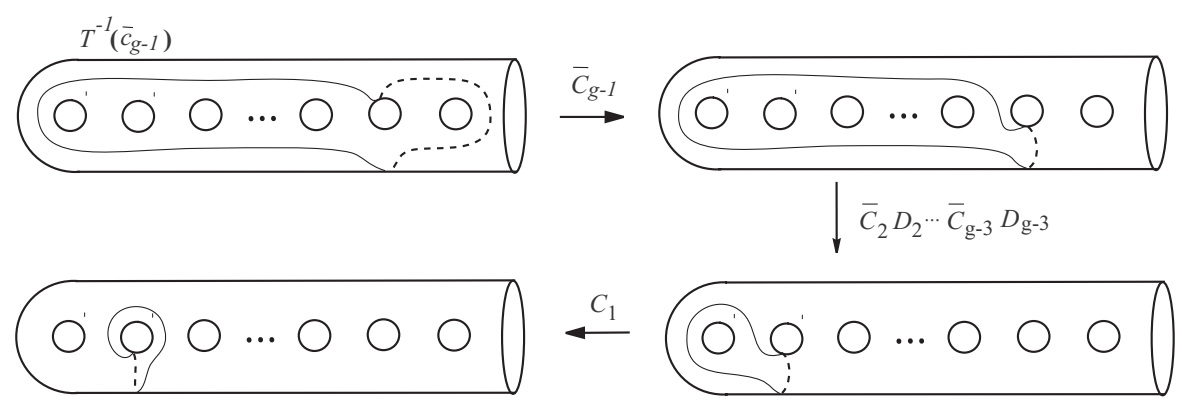

Figure 6 . The proof of $e_{2} \in b^{H}$.

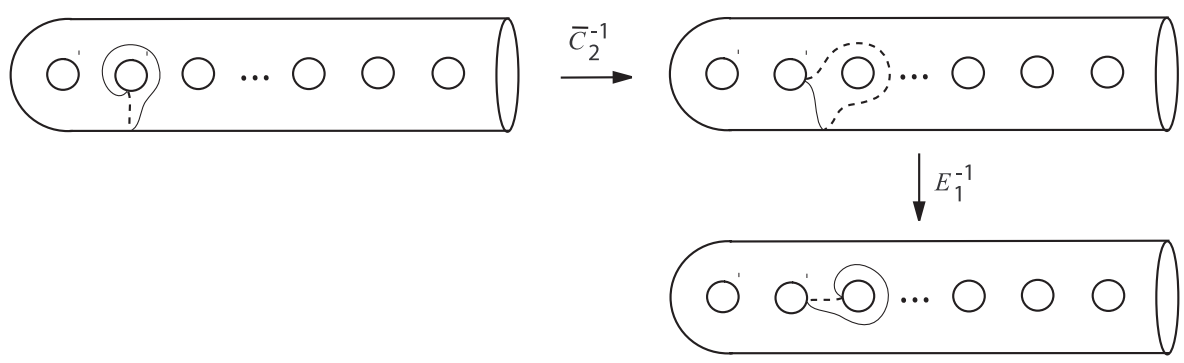

Figure 7. The proof of $e_{3} \in b^{H}$.

The curve $e_{3}$ is the image of the curve $e_{2}$ under the diffeomorphism $E_{1}^{-1}\left(\bar{C}_{2}\right)^{-1}$ (cf. Figure 17). This proves that $e_{3} \in b^{H}$.

Finally, $T^{3}\left(e_{3}\right)=e_{4}$ proving that $e_{4} \in b^{H}$ (cf. Figure 8 ).

Theorem 4.3. The subgroup $H$ generated by $B$ and $T$ is equal to the extended mapping class group $\operatorname{Mod}_{g, 1}^{*}$.

Proof. We prove this theorem in the same way as Theorem [3.2 we show that the $H$-orbit $b^{H}$ of the curve $b$ contains simple closed curves $a_{1}, a_{2}, \ldots, a_{2 g}$. 


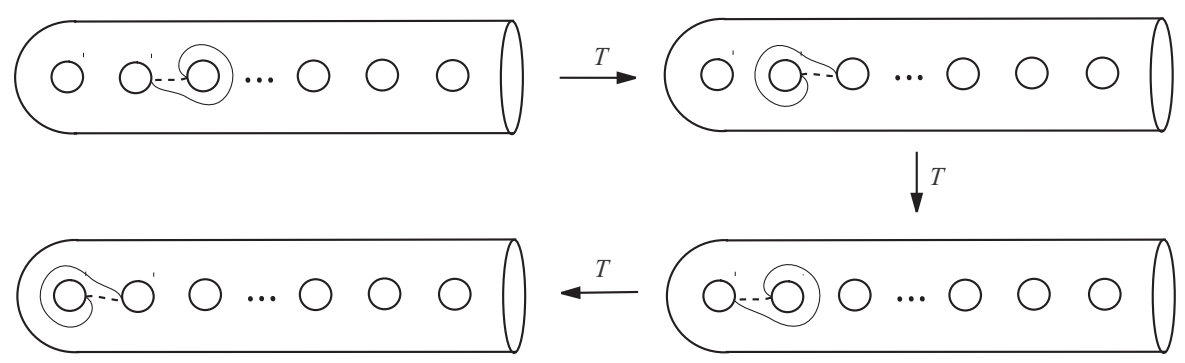

Figure 8. The proof of $e_{4} \in b^{H}$.

It is easy to show that $T\left(a_{i}\right)=a_{i-1}$ for $i=2,3, \ldots, 2 g-1$. Hence, $T A_{i} T^{-1}=$ $A_{i-1}$, and thus $A_{i} \in H$ if and only if $A_{i-1} \in H$.

Suppose that $a_{i_{0}} \in b^{H}$ for some $i_{0}$. Since $B$ is contained in $H$, by Lemma 2.3 we get that $A_{i_{0}}$ is also contained in $H$. Therefore, all $A_{i}$ are contained in $H$. Since $T \in H$, the reflection $R$ is also contained in $H$. The extended mapping class group $\operatorname{Mod}_{g, 1}^{*}$ is generated by the $2 g+1$ Dehn twists $B, A_{1}, A_{2}, \ldots, A_{2 g}$ and the reflection $R$. We conclude that the subgroup $H$ is in fact equal to $\operatorname{Mod}_{g, 1}^{*}$. Therefore, in order to finish the proof of the theorem it suffices to show that $b^{H}$ contains $a_{i}$ for some $i$ with $1 \leq i \leq 2 g$.

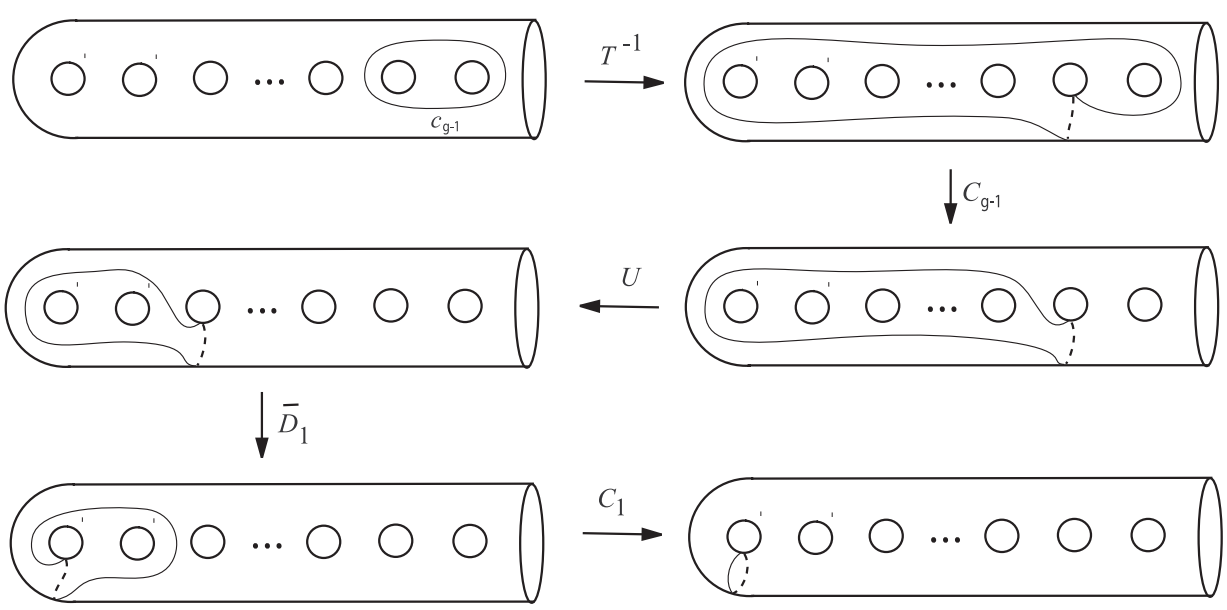

Figure 9. The proof of $a_{1} \in b^{H}$ for even $g$.

Suppose first that $g$ is even. It follows from Lemma 2.3 and Lemma 4.1 that the diffeomorphism

$$
V=C_{1} \bar{D}_{1} C_{3} \bar{D}_{3} \cdots C_{g-3} \bar{D}_{g-3} C_{g-1} T^{-1}
$$

is contained in $H$. Figure 9 shows that $V$ maps the curve $c_{g-1}$ to $a_{1}$. Since $c_{g-1} \in b^{H}, a_{1}$ is also in $b^{H}$. This finishes the proof in this case.

Suppose now that $g$ is odd. It easy to verify that $\left(C_{1}\right)^{-1} E_{4}\left(e_{2}\right)=a_{1}$. Since $e_{2} \in b^{H}$, and $C_{1}$ and $E_{4}$ are in $H$, we conclude that $a_{1} \in b^{H}$.

This completes the proof of the theorem. 


\section{Generating MAPPing Class Groups BY TWO ELEMENTS OF FINITE ORDER}

In this section we prove that the mapping class groups $\operatorname{Mod}_{g, 1}$ and $\operatorname{Mod}_{g}$ are generated by two elements of finite order. Clearly, this will be the minimum number of such generators. In their paper 1], Brendle and Farb proved that these mapping class groups are generated by three torsion elements and asked if they can be generated by two. Therefore our result gives a positive answer to their question.

Let $\Sigma$ be a surface with one boundary component as in Figure 1 . In $\operatorname{Mod}_{g, 1}$, let $S$ denote the product $A_{2 g} A_{2 g-1} \cdots A_{2} A_{1}$. Note that $S$ is of order $4 g+2$. Throughout this section, let $G$ denote the subgroup of $\operatorname{Mod}_{g, 1}$ generated by the two torsion elements $S$ and $B S B^{-1}$. We prove that $G=\operatorname{Mod}_{g, 1}$ for $g \geq 3$.

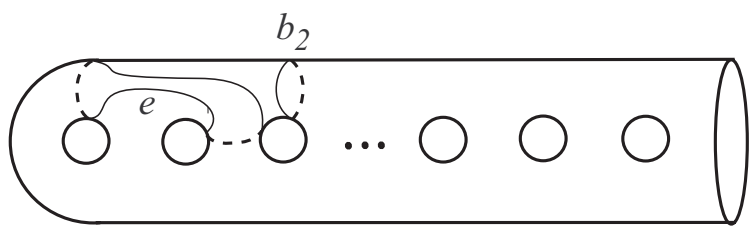

Figure 10. The curves $e$ and $b_{2}$.

In the proof of the main result of this section, we use the celebrated lantern relation, which was discovered by Dehn and rediscovered by Johnson. This relation is read

$$
A_{1} A_{3} A_{5} B_{2}=B D_{1} E,
$$

where the $B_{2}$ and $E$ are the Dehn twists about the curves $b_{2}$ and $e$ in Figure 10. We rewrite the relation as

$$
A_{1}=\left(B A_{3}^{-1}\right)\left(D_{1} A_{5}^{-1}\right)\left(E B_{2}^{-1}\right) .
$$

The strategy of the proof is to show that the statements inside each parenthesis are in $G$. Then the rest of the proof is easy.

Let us define a set $\mathcal{B}$ to be the subset of nonseparating simple closed curves consisting of those curves $x$ such that $X B^{-1} \in G$. That is,

$$
\mathcal{B}=\left\{x \mid x \text { is a nonseparating simple closed curve and } X B^{-1} \in G\right\} .
$$

We first state the following easy to prove, but very useful, lemmas.

Lemma 5.1. Suppose that two simple closed curves $x$ and $y$ are contained in $\mathcal{B}$. Then $X Y^{-1}$ is contained in the subgroup $G$.

Lemma 5.2. Suppose that $y \in \mathcal{B}$ and $X Y^{-1} \in G$. Then $x \in \mathcal{B}$.

Lemma 5.3. Suppose that a curve $y$ is contained in $\mathcal{B}$ and $x$ is in the $\langle S\rangle$-orbit of $y$. Then $x$ is contained in $\mathcal{B}$.

Proof. By assumption, there is an integer $k$ such that $x=S^{k}(y)$. Since the element

$$
X Y^{-1}=\left(S^{k} Y S^{-k}\right) Y^{-1}=S^{k}\left(Y B^{-1}\right)\left(B S B^{-1}\right)^{-k}\left(B Y^{-1}\right)
$$

is contained in $G$, the lemma is proved. 
Corollary 5.4. The curves $c_{i}, \bar{c}_{i}, d_{j}, \bar{d}_{j}$ and $S(b)$ are contained in $\mathcal{B}$ for all $i=$ $1,2, \ldots, g-1$ and $j=1,2, \ldots, g-2$.

Proof. The fact that $c_{i}, d_{j}$ are in the $\langle S\rangle$-orbit of $b$ is shown in Section 3 , It can be shown that $S^{2 g+1}$ is isotopic to the rotation by $\pi$ about the $y$-axis. Since $\bar{c}_{i}=S^{2 g+1}\left(c_{i}\right)$ and $\bar{d}_{j}=S^{2 g+1}\left(d_{j}\right)$, the corollary follows from Lemma 5.3 and the trivial fact that $b \in \mathcal{B}$.

Lemma 5.5. Suppose that $g \geq 3$. Then for each $i=1,2, \ldots, 2 g$, the curves $a_{i}, b_{2}$ and e are contained in $\mathcal{B}$.

Proof. We prove first that the curves $a_{i}$ are contained in $\mathcal{B}$. Since all $a_{i}$ are contained in the same $\langle S\rangle$-orbit, it is enough to prove that any one of $a_{i}$ is in $\mathcal{B}$.

Suppose that $g$ is even, so that $g \geq 4$. Let us denote by $V$ the product $C_{3}^{-1} D_{4}^{-1} \cdots D_{g-4}^{-1} C_{g-3}^{-1} D_{g-2}^{-1} C_{g-1}^{-1}$. We have shown in the proof of Theorem 3.2 that the diffeomorphism $B D_{2}^{-1} V S$ maps the curve $b$ to $a_{4}$. Since the curve $\bar{c}_{1}$ is disjoint from each $c_{i}$ and $d_{j}$ for $i \geq 1, j \geq 3$, the Dehn twist $\bar{C}_{1}$ about $\bar{c}_{1}$ commutes with each $C_{i}$ and $D_{j}$. Therefore it commutes with $V$. Let $x=V S(b)$. Since $\bar{C}_{1} C_{i}^{-1} \in G$ and $\bar{C}_{1} D_{j}^{-1} \in G$, we have $\bar{C}_{1}^{g-3} V \in G$. By the above lemmas, we also have $S B S^{-1} \bar{C}_{1}^{-1} \in G$. Therefore

$$
\begin{aligned}
\left(\bar{C}_{1}^{g-3} V\right)\left(S B S^{-1} \bar{C}_{1}^{-1}\right)\left(V^{-1} \bar{C}_{1}^{3-g}\right) & =(V S) B(V S)^{-1} \bar{C}_{1}^{-1} \\
& =X \bar{C}_{1}^{-1}
\end{aligned}
$$

is contained in $G$. Therefore, $x \in \mathcal{B}$. Moreover, since $B D_{2}^{-1} \in G$ and $X D_{2}^{-1} \in G$, we obtain

$$
\left(B D_{2}^{-1}\right)\left(X D_{2}^{-1}\right)\left(D_{2} B^{-1}\right)=\left(B D_{2}^{-1}\right) X\left(B D_{2}^{-1}\right)^{-1} D_{2}^{-1}=A_{4} D_{2}^{-1}
$$

is contained in $G$. This shows that $a_{4}$, and hence, all $a_{i}$, is in $\mathcal{B}$.

Suppose next that $g$ is odd and $g \geq 5$. Now again let $V$ denote the diffeomorphism $D_{3}^{-1} C_{4}^{-1} D_{5}^{-1} C_{6}^{-1} \cdots D_{g-2}^{-1} C_{g-1}^{-1}$. We have shown in the proof of Theorem 3.2 that the diffeomorphism $C_{2} D_{1} B V S$ maps the the curve $b$ to $a_{3}$. In this case, let $x$ denote the curve $V S(b)$, as above. Equation (5.3) shows that $x \in \mathcal{B}$. We now use the equality

$$
\left(C_{2} D_{1} B C_{4}^{-3}\right)\left(X C_{4}^{-1}\right)\left(C_{2} D_{1} B C_{4}^{-3}\right)^{-1}=A_{3} C_{4}^{-1}
$$

to conclude that $a_{3}$ is in $\mathcal{B}$.

Suppose now that $g=3$. It can easily be shown that $C_{1}^{-1} D_{1}^{-1} S^{2} B S(b)=a_{5}$. We use this fact to prove that $a_{5}$ is contained in $\mathcal{B}$. Notice that $C_{2}^{-1} S(b)=b_{2}$. The equation

$$
\left(\bar{C}_{1} C_{2}^{-1}\right)\left(S B S^{-1} C_{2}^{-1}\right)\left(C_{2} \bar{C}_{1}^{-1}\right)=\left(C_{2}^{-1} S\right) B\left(S^{-1} C_{2}\right) C_{2}^{-1}=B_{2} C_{2}^{-1}
$$

shows that $b_{2} \in \mathcal{B}$. Let $y$ denote the curve $B S(b)$. From the equation

$$
\left(B \bar{C}_{1}^{-1}\right)\left(S B S^{-1} B_{2}^{-1}\right)\left(B \bar{C}_{1}^{-1}\right)^{-1}=Y B_{2}^{-1},
$$

we conclude that $y \in \mathcal{B}$. By Lemma $5.3, z=S^{2}(y)=S^{2} B S(b)$ is also contained in $\mathcal{B}$. Finally, the fact that $a_{5}$ is in $\mathcal{B}$ follows from the equation

$$
\left(C_{1}^{-1} D_{1}^{-1} B_{2}^{2}\right)\left(Z B_{2}^{-1}\right)\left(B_{2}^{-2} D_{1} C_{1}\right)=A_{5} B_{2}^{-1} \text {. }
$$

This concludes the proof of $a_{i} \in \mathcal{B}$. In order to finish the proof of the lemma, it remains to prove that $b_{2}$ and $e$ are contained in $\mathcal{B}$. 
It is easy to see that $C_{2}^{-1} A_{6} A_{5} A_{4}(b)=b_{2}$ and $A_{2} A_{1} A_{4}^{-1} C_{1}\left(a_{5}\right)=e$. It can be shown that the diffeomorphisms $A_{1}^{-2} C_{2}^{-1} A_{6} A_{5} A_{4}$ and $B_{2}^{-2} A_{2} A_{1} A_{4}^{-1} C_{1}$ are in $G$. Finally, $b_{2} \in \mathcal{B}$ follows from

$$
\left(A_{1}^{-2} C_{2}^{-1} A_{6} A_{5} A_{4}\right)\left(B A_{1}^{-1}\right)\left(A_{1}^{-2} C_{2}^{-1} A_{6} A_{5} A_{4}\right)^{-1}=B_{2} A_{1}^{-1},
$$

and $e \in \mathcal{B}$ follows from

$$
\left(B_{2}^{-2} A_{2} A_{1} A_{4}^{-1} C_{1}\right) A_{5} B_{2}^{-1}\left(B_{2}^{-2} A_{2} A_{1} A_{4}^{-1} C_{1}\right)^{-1}=E B_{2}^{-1} .
$$

This completes the proof.

Theorem 5.6. The mapping class group $\operatorname{Mod}_{g, 1}$ (and hence $\operatorname{Mod}_{g}$ ) is generated by two elements of finite order.

Proof. If $g=1$, then $A_{2} A_{1}$ and $A_{1} A_{2} A_{1}$ are of orders 6 and 4 , respectively, and they generate $\operatorname{Mod}_{1,1}$. If $g=2$ then $A_{4} A_{3} A_{2} A_{1}$ and $A_{5} A_{4} A_{3} A_{2} A_{1}$ are of orders 10 and 6 , respectively, and they generate $\operatorname{Mod}_{2,1}$. Suppose that $g \geq 3$ and let $G$ be the subgroup of $\operatorname{Mod}_{g, 1}$ generated by the elements $S=A_{2 g} A_{2 g-1} \cdots A_{2} A_{1}$ and $B S B^{-1}$, which are both of order $4 g+2$. Since the curves $a_{3}, a_{5}, b, b_{2}, d_{1}$ and $e$ are all contained in $\mathcal{B}$, the elements $B A_{3}^{-1}, D_{1} A_{5}^{-1}$ and $E B_{2}^{-1}$ are contained in $G$. By the lantern relation (5.2), $A_{1} \in G$. Therefore, $S^{-i+1} A_{1} S^{i-1}=A_{i} \in G$.

Finally, since the elements $B A_{1}^{-1}$ and $A_{1}$ are in $G, B$ is also in $G$. Consequently, $G=\operatorname{Mod}_{g, 1}$.

This finishes the proof of the theorem.

\section{A presentation of THE MAPPing ClASS GRouP ON TWO GENERATORS}

In this last section we transform the Wajnryb presentation of the mapping class group $\operatorname{Mod}_{g}^{1}$ to a presentatoin on the two generators $B$ and $S$. It turns out that the number of relations in the new presentation depends linearly on $g$, whereas it is quadratic in the original presentation.

Theorem 6.1 ([7, 9]). Let $g \geq 3$. The mapping class group $\operatorname{Mod}_{g}^{1}$ admits a presentation with generators $B, A_{1}, A_{2}, \ldots, A_{2 g}$ and with defining relations

(1) $B A_{i}=A_{i} B$ if $i \neq 4$, and $A_{j} A_{k}=A_{k} A_{j}$ if $|j-k| \geq 2$,

(2) $B A_{4} B=A_{4} B A_{4}, \quad A_{i} A_{i+1} A_{i}=A_{i+1} A_{i} A_{i+1}$ for $1 \leq i \leq 2 g-1$,

(3) $\left(A_{1} A_{2} A_{3}\right)^{4}=B\left(A_{4} A_{3} A_{2} A_{1}^{2} A_{2} A_{3} A_{4}\right) B\left(A_{4} A_{3} A_{2} A_{1}^{2} A_{2} A_{3} A_{4}\right)^{-1}$,

(4) $A_{1} A_{3} A_{5} w B w^{-1}=\left(t_{2} t_{1}\right)^{-1} B\left(t_{2} t_{1}\right) t_{2}^{-1} B t_{2} B$,

where

$t_{1}=A_{2} A_{1} A_{3} A_{2}, t_{2}=A_{4} A_{3} A_{5} A_{4}$ and

$w=A_{6} A_{5} A_{4} A_{3} A_{2}\left(t_{2} A_{6} A_{5}\right)^{-1} B\left(t_{2} A_{6} A_{5}\right)\left(A_{4} A_{3} A_{2} A_{1}\right)^{-1}$.

Suppose that $g \geq 3$. We have shown above that the curve $b$ is mapped to $a_{3}$ by the diffeomorphism

$$
X_{1}=C_{2} D_{1} B D_{3}^{-1} C_{4}^{-1} D_{5}^{-1} C_{6}^{-1} \cdots D_{g-2}^{-1} C_{g-1}^{-1} S
$$

if $g$ is odd, and to $a_{4}$ by

$$
X_{2}=B D_{2}^{-1} C_{3}^{-1} D_{4}^{-1} C_{5}^{-1} \cdots D_{g-2}^{-1} C_{g-1}^{-1} S
$$

if $g$ is even.

From the equalities $S^{-2 k+1}(b)=c_{k}$ and $S^{-2 k}(b)=d_{k}$ we get that

$$
S^{-2 k+1} B S^{2 k-1}=C_{k}
$$


and

$$
S^{-2 k} B S^{2 k}=D_{k},
$$

which should replace $C_{k}$ and $D_{k}$ in (6.1) and (6.2). Also note that $S\left(a_{k}\right)=a_{k-1}$ and hence $S A_{k} S^{-1}=A_{k-1}$. Let us now define

$$
X= \begin{cases}S^{3} X_{1} & \text { if } g \text { is odd, } \\ S^{4} X_{2} & \text { if } g \text { is even, }\end{cases}
$$

so that $S^{-k} X(b)=a_{k}$, and therefore

$$
A_{k}=S^{-k} X B X^{-1} S^{k}=B^{S^{-k} X}
$$

for every $k=1,2, \ldots, 2 g$.

The presentation of the mapping class group $\operatorname{Mod}_{g}^{1}$ in Theorem 6.1 has $2 g+1$ generators and $2 g^{2}+g+2$ relations. By replacing each $A_{k}$ in Theorem 6.1 by $S^{-k} X B X^{-1} S^{k}$, we get another presentation of $\operatorname{Mod}_{g}^{1}$ on the two generators $B$ and $S$. Now these relations are not as nice as those of Wajnryb's, but the number of relations in the new presentation reduces to $4 g+2$.

For any $i, j$ with $|i-j|>1$ and for any $k$, all the relations $A_{i+k} A_{j+k}=A_{j+k} A_{i+k}$ reduce to the single relation $B^{X} B^{S^{i-j} X}=B^{S^{i-j} X} B^{X}$. This shows that the relations in (1) give rise to $(2 g-1)+(2 g-2)=4 g-3$ relations.

Each braid relation $A_{k} A_{k+1} A_{k}=A_{k+1} A_{k} A_{k+1}$ reduces to the single relation $B^{X} B^{S^{-1} X} B^{X}=B^{S^{-1} X} B^{X} B^{S^{-1} X}$. Thus the relations in (2) reduce to two relations.

One more relation comes from the equality $S=A_{2 q} A_{2 q-1} \cdots A_{2} A_{1}$.

Consequently the new presentation of $\operatorname{Mod}_{g}^{1}$ is given as follows: Let $P$ denote $B^{X}$ and let $U$ denote $S^{-1}$, where $X$ is defined as in (6.3).

Theorem 6.2. Let $g \geq 3$. The mapping class group $\operatorname{Mod}_{g}^{1}$ admits a presentation with generators $B$ and $U$, and with defining relations

(1) $B P^{U^{i}}=P^{U^{i}} B$, for $1 \leq i \leq 2 g$ and $i \neq 4$,

(2) $P P^{U^{i}}=P^{U^{i}} P$ for $2 \leq i \leq 2 g-1$,

(3) $B P^{U^{4}} B=P^{U^{4}} B P^{U^{4}}$ and $P P^{U} P=P^{U} P P^{U}$,

(4) $\left(P^{U} P^{U^{2}} P^{U^{3}}\right)^{4}=B B^{V}$, and

(5) $P^{U} P^{U^{3}} P^{U^{5}} B^{W}=B B^{t_{1}^{-1} t_{2}^{-1}} B^{t_{2}^{-1}}$,

(6) $U P^{U^{2 g}} P^{U^{2 g-1}} \cdots P^{U^{2}} P^{U}=1$,

where

$V=P^{U^{4}} P^{U^{3}} P^{U^{2}} P^{U} P^{U} P^{U^{2}} P^{U^{3}} P^{U^{4}}$,

$t_{1}=P^{U^{2}} P^{U} P^{U^{3}} P^{U^{2}}, t_{2}=P^{U^{4}} P^{U^{3}} P^{U^{5}} P^{U^{4}}$ and

$W=P^{U^{6}} P^{U^{5}} P^{U^{4}} P^{U^{3}} P^{U^{2}}\left(t_{2} P^{U^{6}} P^{U^{5}}\right)^{-1} B\left(t_{2} P^{U^{6}} P^{U^{5}}\right)\left(P^{U^{4}} P^{U^{3}} P^{U^{2}} P^{U}\right)^{-1}$.

The presentation of the mapping class group $\operatorname{Mod}_{g}$ is obtained from that of $\operatorname{Mod}_{g}^{1}$ by adding one more relation (cf. 9]). Hence, in the new presentation on the generators $B$ and $S$ the number of relations reduces to $4 g+2$.

\section{ACKNOWLEDGMENT}

The author is grateful to Nathan Dunfield for pointing out that the relation in Theorem 6.2 (6) was missing in the earlier version of the paper. 


\section{REFERENCES}

1. T. Brendle, B. Farb, Every mapping class group is generated by 6 involutions, J. Algebra 278 (2004), 187-198. MR2068073

2. M. Dehn, Die gruppe der abdildungsklassen, Acta Math. 69 (1938), 135-206.

3. S. Humphries, Generators for the mapping class group, in: Topology of Low Dimensional Manifolds, Ed. by R. Fenn, Lecture Notes in Math. No. 722, Springer-Verlag, Berlin, 1979, 44-47. MR80i:57010

4. D. L. Johnson, The structure of the Torelli group I: A finite set of generators for $\mathcal{I}$, Annals of Math. (2) 118 (1983), 423-442. MR85a:57005

5. W. B. R. Lickorish, A representation of orientable combinatorial 3-manifolds, Annals of Mathematics 76 (1962), 531-540. MF 27:1929

6. W. B. R. Lickorish, A finite set of generators for the homeotopy group of a 2-manifold, Math. Proc. Camb. Phil. Soc. 60 (1964), 769-778. MR30:1500

7. B. Wajnryb, A simple presentation for the mapping class group of an orientable surface, Israel Jounal of Mathematics 45 (1983), 157-174. MR.85g:57007

8. B. Wajnryb, Mapping class group of a surface is generated by two elements, Topology 35 (1996), 377-383. MR96m:57007

9. B. Wajnryb, An elementary approach to the mapping class group of a surface, Geom. Topol. 3 (1999), 405-466. MR2001a:20059

Department of Mathematics, Middle East Technical University, 06531 Ankara, TURKEY

E-mail address: korkmaz@arf.math.metu.edu.tr 